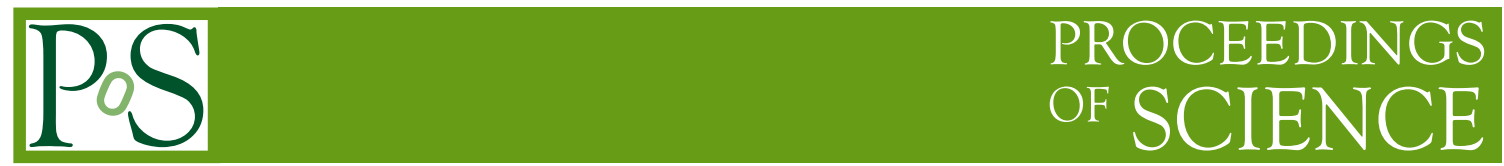

\title{
New results of the digital radio interferometer LOPES
}

F.G. Schröder*1, K. Link ${ }^{2}$, W.D. Apel ${ }^{1}$, J.C. Arteaga-Velázquez ${ }^{3}$, L. Bähren ${ }^{4}$, K. Bekk ${ }^{1}$, M. Bertaina ${ }^{5}$, P.L. Biermann ${ }^{5,1}$, J. Blümer ${ }^{1,6}$, H. Bozdog ${ }^{1}$, I.M. Brancus ${ }^{7}$, E. Cantoni ${ }^{4,8}$, A. Chiavassa ${ }^{5}$, K. Daumiller ${ }^{1}$, V. de Souza ${ }^{9}$, F. Di Pierro ${ }^{5}$, P. Doll ${ }^{1}$, R. Engel ${ }^{1}$, H. Falcke ${ }^{10,3,5}$, B. Fuchs ${ }^{2}$, H. Gemmeke ${ }^{11}$, C. Grupen ${ }^{12}$, A. Haungs ${ }^{1}$, D. Heck ${ }^{1}$, J.R. Hörandel ${ }^{10}$, A. Horneffer ${ }^{6}$, D. Huber ${ }^{2}$, T. Huege ${ }^{1}$, P.G. Isar ${ }^{13}$, K-H. Kampert ${ }^{14}$, D. Kang ${ }^{2}$, O. Krömer ${ }^{11}$, J. Kuijpers ${ }^{10}$, P. Łuczak ${ }^{15}$, M. Ludwig ${ }^{2}$, H.J. Mathes ${ }^{1}$, M. Melissas ${ }^{2}$, C. Morello ${ }^{8}$, J. Oehlschläger ${ }^{1}$, N. Palmieri ${ }^{2}$, T. Pierog ${ }^{1}$, J. Rautenberg ${ }^{14}$, H. Rebel ${ }^{1}$, M. Roth ${ }^{1}$, C. Rühle ${ }^{11}$, A. Saftoiu ${ }^{7}$, H. Schieler ${ }^{1}$, A. Schmidt ${ }^{11}$, S. Schoo ${ }^{1}$, O. Sima ${ }^{16}$, G. Toma ${ }^{7}$, G.C. Trinchero ${ }^{8}$, A. Weindl ${ }^{1}$, J. Wochele ${ }^{1}$, J. Zabierowski ${ }^{15}$, J.A. Zensus ${ }^{5}$ - LOPES Collaboration

${ }^{1}$ Institut für Kernphysik, Karlsruher Institut für Technologie (KIT), Germany

${ }^{2}$ Institut für Experimentelle Kernphysik, Karlsruher Institut für Technologie (KIT), Germany

${ }^{3}$ Instituto de Física y Matemáticas, Universidad Michoacana, Morelia, Mexico

${ }^{4}$ ASTRON, Dwingeloo, The Netherlands

${ }^{5}$ Dipartimento di Fisica, Università degli Studi di Torino, Torino, Italy

${ }^{6}$ Max-Planck-Institut für Radioastronomie, Bonn, Germany

${ }^{7}$ National Institute of Physics and Nuclear Engineering, Bucharest-Magurele, Romania

${ }^{8}$ Osservatorio Astrofisico di Torino, INAF Torino, Italy

${ }^{9}$ Universidade São Paulo, Instituto de Física de São Carlos, São Carlos, Brasil

${ }^{10}$ Department of Astrophysics, Radboud University Nijmegen, The Netherlands

${ }^{11}$ Institut für Prozessdatenverarbeitung und Elektronik, KIT, Germany

${ }^{12}$ Faculty of Natural Sciences and Engineering, Universität Siegen, Germany

${ }^{13}$ Institute for Space Sciences, Bucharest-Magurele, Romania

${ }^{14}$ Fachbereich C, Physik, Universität Wuppertal, Germany

${ }^{15}$ Department of Astrophysics, National Centre for Nuclear Research, Eódź, Poland

${ }^{16}$ Department of Physics, University of Bucharest, Bucharest, Romania

E-mail: frank.schroederekit.edu

The 34th International Cosmic Ray Conference,

30 July- 6 August, 2015

The Hague, The Netherlands

\footnotetext{
* Speaker.
} 


\begin{abstract}
LOPES was a digital, phased antenna array located at the site of KASCADE-Grande in Karlsruhe, Germany, operating from 2003 to 2013 . Triggered by the particle detector array of KASCADE, LOPES measured the radio signal of air showers between 40 and $80 \mathrm{MHz}$ (effective bandwidth: $43-74 \mathrm{MHz}$ ). By cross-correlation beamforming, i.e., an interferometric, offline combination of the signals measured at different antennas, LOPES was able to enhance the signal-to-noise ratio. This lowered the detection threshold significantly close to $0.1 \mathrm{EeV}$, despite the high human-made radio background at the experimental site. Although LOPES already has been dismantled, data analysis is still continued. Recent progress concerns a new amplitude calibration of the existing data, described in the second proceeding, and the incorporation of detector effects in air-shower simulations produced with CoREAS. These end-to-end simulations enable a better comparison of measurements with theoretical predictions. Furthermore, the new end-to-end simulations have been used to study the effect of the antenna and the bandpass filter on the slope of the lateral distribution and the shape of the wavefront. Both parameters can be used to reconstruct the shower maximum independently: the reconstruction of the lateral distribution relies on amplitude measurements, and the reconstruction of the hyperbolic radio wavefront relies on time measurements. In the new end-to-end simulations, the lateral distributions are slightly steeper than in the previously used simplified simulations. For the wavefront we do not find a significant change, but with both methods, the uncertainties are still large due to the high background at the LOPES site. Nevertheless, with the end-to-end simulations we do find a large influence of the used method for reconstruction of the hyperbolic wavefront: Fitting pulse arrival times in individual antennas, the reconstructed cone angle of the wavefront is on average half of the angle determined by cross-correlation beamforming. This makes a quantitative comparison between different experiments and to simulations more difficult, since all details of the wavefront reconstruction have to be considered appropriately. Nevertheless, the two effects on the wavefront reconstruction (cross-correlation beamforming vs. arrival time fit; simplified vs. end-to-end simulations) on average cancel out, such that previously published LOPES results remain valid. In particular the cone angle $\rho$ of the wavefront predicted by CoREAS is compatible with the measured one.
\end{abstract}




\section{Introduction}

LOPES was the radio extension of the KASCADE-Grande experiment, in Karlsruhe, Germany [1, 2]. It was built in 2003 as LOFAR prototype station for the proof-of-principle that air showers can be detected by digital radio interferometry [3]. Beginning of 2013, the operation of LOPES was stopped together with the operation of the KASCADE-Grande host experiment. Both experiments meanwhile have been dismantled. Nevertheless, data analysis still continues, and in future LOPES data are planned to be made publicly available within $\operatorname{KCDC}[4,5]$.

Since the last ICRC in 2013, two papers have been published on the amplitude and time characteristics of the measured radio signal, and the application for the reconstruction of air-shower properties. The lateral distribution of the radio amplitudes can be used to reconstruct the energy and the position of the shower maximum $[6,7]$. The shower maximum can also be reconstructed from the radio wavefront [8], which itself is reconstructed based on the arrival time of the signal. According to our analysis, the radio wavefront has approximately hyperbolic shape, which soon was confirmed by LOFAR measurements of better quality [9].

Moreover, we have new results on the amplitude calibration of LOPES. As described in our second proceeding [10], a re-calibration of our reference source used for the amplitude calibration lowered the scale of all LOPES amplitude measurements by a factor of $2.6 \pm 0.2$ [11]. Thus, the measured radio amplitude now is compatible with CoREAS simulations, but not any longer with REAS 3.11 simulations as published earlier [12]. Other results remain qualitatively valid.

Finally, we have improved the method of comparing simulations to measurements, now performing end-to-end simulations which incorporate all known properties of the LOPES detector, instead of just the most important features. This proceeding focuses on the results of this new simulation study and its impact on the interpretation of LOPES measurements.

\section{Experimental configuration and data selection}

As technical demonstrator different antenna types and array configurations were tested. The latest results on the reconstruction of the energy and the shower maximum were obtained with eastwest aligned, inverted v-shape dipole antennas. These antennas have been triggered by KASCADEGrande, and measured the radio signal in an effective bandwidth of $43-74 \mathrm{MHz}$. Results of the other LOPES setups can be found in corresponding references, e.g., on vectorial measurements with three orthogonally crossed dipoles [13].

Generally the analysis of LOPES measurements consists of several steps. As first step, events with zenith angle $\theta<45^{\circ}$ and energy $E>10^{17} \mathrm{eV}$ as reconstructed by KASCADE-Grande are selected. As second step, we apply a digital, interferometric technique, namely cross-correlation beamforming. The digitally recorded radio traces are shifted in time according to the arrival direction of the air-shower reconstructed by KASCADE-Grande. Then, the maximum of a crosscorrelation of all antenna traces is determined in a region around this initial direction, leading to an average deviation between the KASCADE-Grande and LOPES directions of less than $0.7^{\circ}$. Moreover, the steepness of the hyperbolic wavefront is varied maximizing the cross-correlation amplitude. The resulting average angle $\rho$ of the asymptotic cone to the shower plane is $(1.2 \pm 0.3)^{\circ}$ [9]. This procedure of interferometric cross-correlation beamforming lowers the detection threshold of 


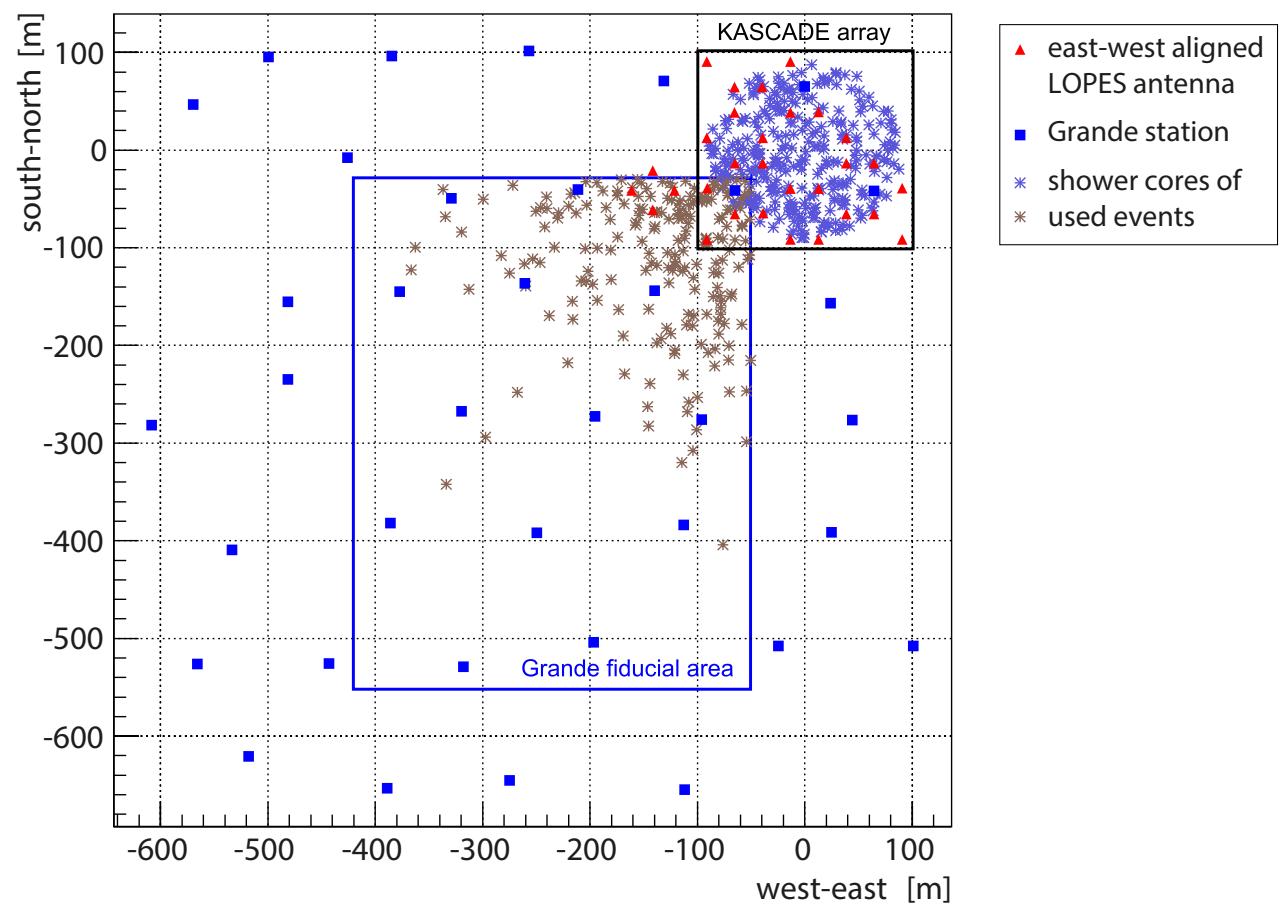

Figure 1: Map of the LOPES and KASCADE-Grande experiments. Shower cores of LOPES events passing signal-to-noise and quality cuts are indicated as stars. For the results presented in this proceeding only the events with core inside the circular fiducial area of KASCADE are used.

LOPES, which is essential for the operation in a radio-loud environment. The interferometric technique is only possible due to a nanosecond-level accurate relative timing [14]. Finally, in a third step after the air-shower pulse has been identified by beamforming, its arrival time and amplitude is determined in each individual antenna.

Depending on the exact quality cuts, about 500 air-shower events are used for different analyses. About 300 of these events have the shower core inside the fiducial area of the KASCADE particle detector array, thus, also inside the LOPES antenna array (see figure 1). This provides a higher reconstruction quality, and only those events are used for the wavefront and the shower maximum analyses, while for the comparison to simulations all events are used.

Complementing the measurements, we produced two CORSIKA [15] Monte-Carlo simulations for each event, one for a proton, and one for an iron nucleus as primary particle, using the direction, core, and energy reconstructed by KASCADE-Grande as initial parameters. The radio emission is calculated by CoREAS [16, 17], which includes all emission processes known to be relevant, in particular the geomagnetic deflection of electrons and positrons, the Askaryan effect, i.e., radio emission due to the variation of the net charge of the shower front, and Cherenkov-like effects due to the refractive index of air. These simulations are compared to LOPES measurements to test the model. They are also used to develop and calibrate the reconstruction of the shower maximum based on the slope of the lateral distribution and on the cone angle of the wavefront. Due to the different distance of the shower maximum to the detector proton initiated showers on average have a steeper lateral distribution, and a larger cone angle $\rho$ of the wavefront than iron showers. 

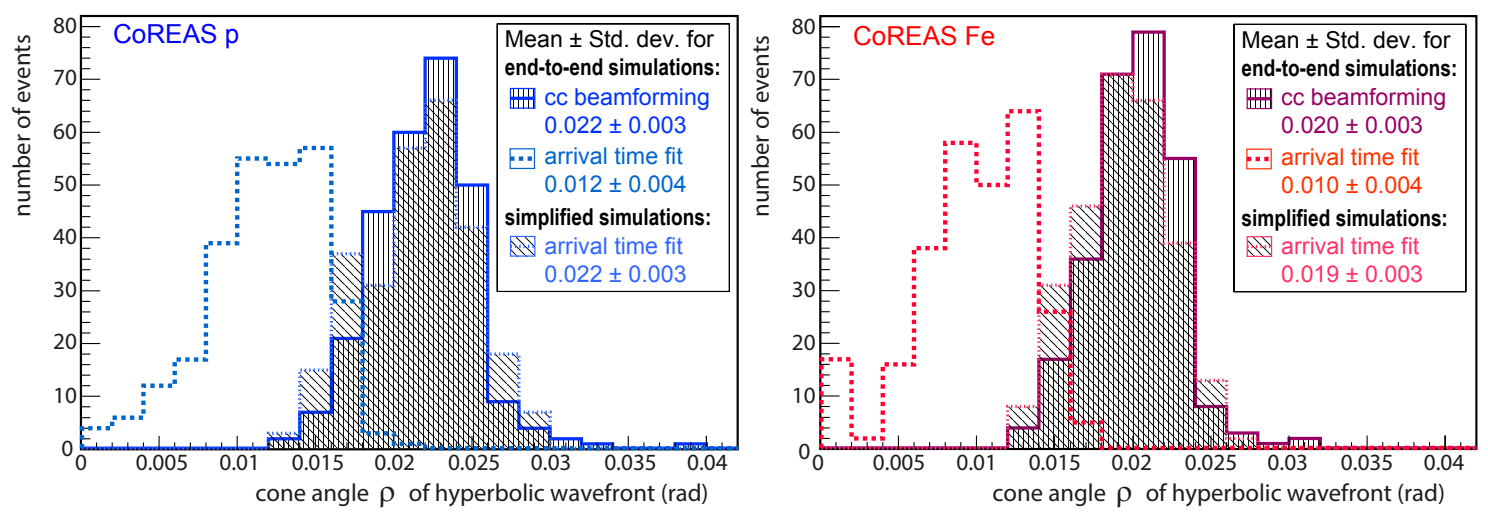

Figure 2: Change of the cone angle $\rho$ of the wavefront depending on the reconstruction method for the CoREAS simulations with proton and iron nuclei as primary particles. When reconstructed by crosscorrelation beamforming (as in the LOPES measurements) $\rho$ is approximately twice as large in the endto-end simulations as by fitting the arrival times in the individual antennas. The old method of simplified simulations used in reference [8], which does not correct for pulse distortion by the antenna and the bandpass filter, roughly gives the same value as cross-correlation beamforming in the new end-to-end simulations.

For previously published results we did not perform end-to-end simulations including all detector effects. Instead, we simply filtered the east-west polarization component of the simulations to the measured bandwidth of $43-74 \mathrm{MHz}$. Effectively this means that the response pattern of the antenna, the signal distortion in the bandpass filter, and possible non-linear effects introduced by the cross-correlation beamforming are not taken into account. Nevertheless, as shown earlier [18], this is a sufficient approximation when comparing measured and simulated amplitudes, since on average this leads to a small mistake, only, compared to the measurement uncertainties.

The newly available end-to-end simulations are based on the same CoREAS simulations treated earlier in a simplified way. Now, we apply the response pattern of the LOPES antenna on the simulated electric field as well as the known signal-changing properties of the amplifiers and bandpass filter, and quantize the signal according to the used ADCs. By this procedure we obtain an artificial event, which afterwards is processed in the same way as a normally measured LOPES event. The main remaining difference to measured events is that we have not yet added background in the simulated events.

We studied the effect of the end-to-end simulations on the cone angle of the wavefront, and the slope of the lateral distribution, i.e., the parameters used for the reconstruction of the shower maximum. Also here the absolute effect is small, but since the atmospheric depths of the shower maxima of proton and iron showers on average only differ by $20 \%$, even small changes in the parameters can significantly affect the interpretation. The results are still preliminary, since we have not yet studied the effect of background on the end-to-end simulations. Since most of the LOPES measurements are close to the threshold, we cannot exclude a systematic bias.

\section{Results}

\subsection{Wavefront (arrival times)}

For the hyperbolic wavefront we determined the angle $\rho$ between the asymptotic cone of the 

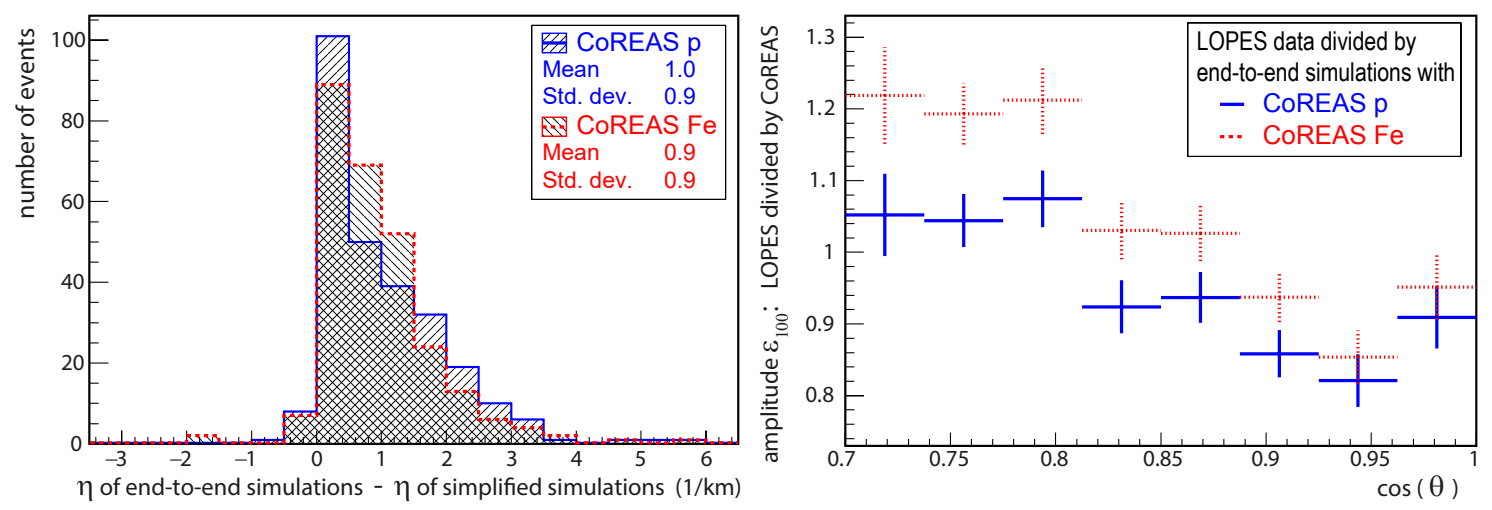

Figure 3: Left: comparison of the lateral slope parameter $\eta$ of the simplified evaluation of CoREAS simulations used before with the new end-to-end simulations including all detector effects known to be relevant, except of noise. Right: profile (= bins with mean and standard deviation) of the ratio between simulated and measured amplitudes over the zenith angle $\theta$ ( 8 events which are clear outliers in the comparison of measured and simulated amplitude $\varepsilon_{100}$ [10] have been removed for this plot. They do not change the trend, but would dominate the standard deviation.)

hyperbola and the shower plane, and fixed the offset in the apex to $3 \mathrm{~ns}$, exactly as in reference [8]. However, for the LOPES measurements and the simulations we previously determined $\rho$ in different ways: For the measurements we maximized the amplitude of the cross-correlation, for the simulations we fitted the wavefront to the maximum time of the pulse in each individual antenna. The end-to-end simulations reveal that the pulse distortion of the antenna and the filters have significant impact: The cone angle $\rho$ is on average only half as large compared to the simplified simulations not taking into account these detector effects (see figure 2).

We now were able to compare also the systematic difference between the two reconstruction methods: the cone angle $\rho$ determined by cross-correlation beamforming is approximately twice as large as the one determined by fitting individual maximum times. Interestingly, this means that both effects (reconstruction method and pulse distortion) compensate each other within uncertainties, and only for 15 out of 276 simulated events the deviation is larger than $0.3^{\circ}$. On average the value of $\rho$ determined with the arrival-time fit to the simplified simulations matches the one determined by cross-correlation beamforming with the end-to-end simulations, and both are compatible with the average cone angle measured by LOPES. ${ }^{1}$

For the wavefront analysis, it is not yet clear, if a small shift in the reconstructed $X_{\max }$ values will occur. In reference [8], we used the following formula for $X_{\max }$ reconstruction: $X_{\max }=c \cdot \rho$. $\cos ^{-\gamma}(\theta)$ (with $\rho$ given in radians). For the end-to-end simulations the proportionality constant $c$ will change slightly from about 25,000 to $26,000 \mathrm{~g} / \mathrm{cm}^{2}$, and the zenith dependence of $\rho$ becomes smaller, changing $\gamma$ from approximately 1.5 to 1.1 . As net effect, no significant change in the mean reconstructed $X_{\max }$ occurs in the simulations.

\subsection{Lateral distribution of amplitudes}

For the lateral distribution, in earlier analyses $[6,12]$ we fitted a simple exponential function for

\footnotetext{
${ }^{1}$ If unknown effects lead to a discrepancy, it is only natural to look intensively for a reason. Only self-compensating effects - for whatever reason - remain. This enhances the risk for self-compensating effects above the chance level.
} 
the amplitude $\varepsilon$ as function of the distance to the shower axis $d: \varepsilon(d)=\varepsilon_{100} \exp [-\eta(d-100 \mathrm{~m})]$, with two fit parameters, namely the amplitude $\varepsilon_{100}$ at $100 \mathrm{~m}$ axis distance, and a slope parameter $\eta$, which on average was $(6 \pm 4) \mathrm{km}^{-1}$ for the LOPES measurements. This lateral distribution function, neglects the azimuthal asymmetry of the radio footprint due to the Askaryan effect [21, 19, 20], and the peak around $100 \mathrm{~m}$ axis distance due to Cherenkov-like effects [22]. Though the latter one was taken into account by a Gaussian function used in reference [7], until now, we only have repeated the analyses with the exponential function using the new end-to-end simulations.

Figure 3 (left), shows the average effect of the end-to-end simulations on the slope of the lateral distribution. On average the lateral distribution becomes steeper, i.e., $\eta$ increases by $(1 \pm 0.9) \mathrm{km}^{-1}$. The measured distribution of $\eta$ still is in-between the simulated distributions for a pure proton and a pure iron composition, but the measured data now are closer to the iron simulations. The effect on the $X_{\max }$ reconstruction presented in reference [7] based on a Gaussian LDF has to be investigated in more detail.

Finally, we used the new end-to-end simulations to compare the measured amplitudes with the one simulated by CoREAS. In reference [7], we noted for the simplified simulations that measured and simulated amplitudes depend differently on zenith angle. As already for the simplified simulations [12], all other studied dependencies of $\eta$ and $\varepsilon_{100}$ are consistently described by CoREAS. We now studied the zenith discrepancy with the end-to-end simulations, again (figure 3 , right): it can neither be explained by the re-calibration, nor by the missing detector effects in the simplified simulations, although the discrepancy is slightly weaker for the end-to-end simulations than for the simplified simulations [10]. However, we cannot exclude that the zenith dependence of the used antenna model can be inaccurate on this level of precision. Since LOPES is already dismantled, it is difficult to perform further calibration measurements to solve this issue. Thus, it has to be checked by other experiments.

\section{Conclusion}

There are two major, independent improvements in the LOPES analyses: first, a new calibration of the amplitude scale described in reference [10]; second the availability of a software to apply relevant detector effects to CoREAS simulations of the radio emission by air showers. Using the end-to-end simulations we observe that the lateral distribution of the radio amplitude becomes systematically steeper due to the detector effects now taken into account (like pulse distortion by the antenna and the bandpass filter) - at least when using a simple exponential LDF. Other results do not change qualitatively. In particular, the direction resolution of better than $0.7^{\circ}$, and the energy precision of at least $20 \%[7,23]$ are not affected.

Moreover, with the end-to-end simulations we discovered that the method for reconstructing the wavefront shape has significant impact on the resulting cone angle $\rho$ of the wavefront, as the pulse distortion by the signal chain (antenna, bandpass filter, ...) has. After taking into account these detector effects, the cone angle determined by cross-correlation beamforming is approximately twice as large as the one determined by fitting arrival times in individual antennas. This has to be taken into account when comparing wavefront measurements by LOPES with other experiments, as well as a possible bias due to noise. For the LOPES results on the wavefront published in reference [8], nevertheless, the impact is negligible: the two strong effects, namely the change of 
$\rho$ between cross-correlation beamforming and arrival time fitting, and the change of $\rho$ due to the pulse distortion, on average cancel out. Consequently, also in the new analysis the radio wavefront predicted by CoREAS is compatible to the measured one.

\section{Acknowledgement}

LOPES and KASCADE-Grande have been supported by the German Federal Ministry of Education and Research. KASCADE-Grande is partly supported by the MIUR and INAF of Italy, the Polish Ministry of Science and Higher Education and by the Romanian Authority for Scientific Research UEFISCDI (PNIIIDEI grant 271/2011). This research has been supported by grant number VH-NG-413 of the Helmholtz Association, and BMBF Verbundforschung. The present study is supported by the 'Helmholtz Alliance for Astroparticle Physics - HAP' funded by the Initiative and Networking Fund of the Helmholtz Association, Germany.

\section{References}

[1] T. Antoni et al. (KASCADE Coll.): Nucl. Instr. Meth. A 513 (2003) 490

[2] W.D. Apel et al. (KASCADE-Grande Coll.): Nucl. Instr. Meth. A 620 (2010) 202

[3] H. Falcke et al. (LOPES Coll.): Nature 435 (2005) 313

[4] A. Haungs et al. (KASCADE-Grande Coll.), Journal of Phys.: Conf. Ser. (JPCS) 632 (2015) 012011

[5] S. Schoo et al. (KASCADE-Grande Coll.): this issue, PoS (ICRC2015) 262

[6] W.D. Apel et al. (LOPES Coll.): Phys. Rev. D 85 (2012) 071101(R)

[7] W.D. Apel et al. (LOPES Coll.): Phys. Rev. D 90 (2014) 062001

[8] W.D. Apel et al. (LOPES Coll.): J. Cosmol. Astropart. Phys. 09 (2014) 025

[9] A. Corstanje et al. (LOFAR Coll.): Astropart. Phys. 61 (2015) 22

[10] K. Link et al. (LOPES Coll.): this issue, PoS (ICRC2015) 311

[11] W.D. Apel et al. (LOPES Coll.): Astropart. Phys. 75 (2016) 72

[12] W.D. Apel et al. (LOPES Coll.): Astropart. Phys. 50-52 (2013) 76

[13] W.D. Apel et al. (LOPES Coll.): Nucl. Instr. Meth. A 696 (2012) 100

[14] F.G. Schröder, et al.: Nucl. Instr. Meth. A 615 (2010) 277

[15] D. Heck, et al.: FZKA Report 6019 Forschungszentrum Karlsruhe 1998

[16] M. Ludwig, T. Huege: Astropart. Phys. 34 (2011) 438

[17] T. Huege, et al.: AIP Conf. Proc. 1535 (2013) 128

[18] K. Link et al. (LOPES Coll.): Proc. of ICRC 2013, Rio de Janeiro, Brazil (2013) number 0844

[19] Pierre Auger Coll.: Phys. Rev. D 89 (2014) 052002

[20] P. Schellart, et al. (LOFAR Coll.): J. Cosmol. Astropart. Phys. 10 (2014) 01

[21] A. Belletoile, et al. (CODALEMA Coll.): Astropart. Phys. 69 (2015) 50

[22] A. Nelles, et al. (LOFAR Coll.): Astropart. Phys. 65 (2014) 11

[23] F.G. Schröder et al. (LOPES Coll.): Journal of Phys.: Conf. Ser. (JPCS) 632 (2015) 012102 